\title{
Quantum Manipulation and Measurement of Single Atoms in Optical Cavity QED
}

\author{
Jun Ye, Christina J. Hood, Theresa Lynn, Hideo Mabuchi, David W. Vernooy, and H. Jeff Kimble
}

\begin{abstract}
Using laser-cooled atoms strongly coupled to a high finesse optical cavity, we have performed real-time continuous measurements of single atomic trajectories in terms of the interaction energy $\left(E_{\text {int }}\right)$ with the cavity. Individual transit events reveal a shot-noise limited measurement (fractional) sensitivity of $4 \times 10^{-4} / \sqrt{\mathrm{Hz}}$ to variations in $E_{\text {int }} / \hbar$ within a bandwidth of 1-300 kHz. The strong coupling of atom and cavity leads to a maximum interaction energy greater than the kinetic energy of an intracavity laser-cooled atom, even under weak cavity excitation. Evidence of mechanical light forces for intracavity photon number $<1$ has been observed. The quantum character of the nonlinear optical response of the atom-cavity system is manifested for the trajectory of a single atom.
\end{abstract}

Index Terms - Atomic physics, cavity QED, cooling and trapping, measurement, quantum measurement, real-time observation, strong coupling.

\section{INTRODUCTION}

O PTICAL cavity quantum electrodynamics (QED) in the strong coupling regime [1] plays a significant role in exploring manifestly quantum dynamics where coherent, reversible system evolution dominates over dissipative processes. The large coherent-interaction rate generates a unique capability of monitoring quantum processes in real time, which in turn should lead eventually to the investigation of the strong conditioning of system evolution on measurement results and the realization of quantum feedback control [2]. Another important feature associated with strong coupling is that system dynamics are readily influenced by single quanta. Thus single-atom and single-photon cavity QED provides an ideal stage where the dynamical processes of individual quantum systems can be isolated and manipulated. Such coherently controlled processes are essential to advances of quantum information technologies.

The dipole coupling rate $g_{0}$ between a two-level atom and a small, high-finesse cavity sets the rate of energy exchange between the two constituents. This internal evolution of the composite system is accompanied by two dissipation channels, namely the cavity decay rate $\kappa$ and the free-space atomic decay $\gamma_{\perp}$. However, $\kappa$ can also be regarded as providing a quantum channel through which the information of the open quantum system can be extracted, processed, and fed back with high efficiency. The other relevant time scale, the interaction time $T$, is associated with the center-of-mass (CM)

Manuscript received July 2, 1998. This work was supported by the National Science Foundation, the Office of Naval Research, and DARPA via the QUIC Institute administered by ARO.

The authors are with the Norman Bridge Laboratory of Physics California Institute of Technology, Pasadena, CA 91125 USA.

Publisher Item Identifier S 0018-9456(99)02923-X. motion (external degree-of-freedom) of the atom through the spatially-varying cavity eigenmode. The use of laser-cooled atoms in cavity QED [3] affects a dramatic separation of dynamical time scales, with $g_{0}>\kappa, \gamma_{\perp} \gg 1 / T$. The rate of coherent coupling between the atom and cavity, $g_{0}^{2} / \kappa$, sets the maximum information bandwidth of the intracavity activity. The optical information per atomic transit is therefore $g_{0}^{2} T / \kappa \gg 1$, eliminating the need to base measurements on averages over an ensemble of atom transits.

In this paper, we describe two experiments that explore phenomena uniquely accessible in this regime of true strong coupling. In the first part we investigate the real-time continuous quantum measurement of the interaction energy between the atom and cavity during individual transit events [4]. Within a broader context, we are actively exploring the issue of how the dynamical behavior of a continuously-observed open quantum system is conditioned [5], [6] upon the measurement record, which in our case is the broadband photocurrent resulting from cavity leakage photons. In the second part we study the mechanical force on single atoms brought by single intracavity photons via the strong coupling [7]. Laser cooled atoms have sufficiently low kinetic energies that a single quantum (excitation of the composite atom-cavity system) is able to affect profoundly the atomic CM motion.

\section{Continuous Measurement of Single-Atom Dynamics}

The cavity we used in this experiment was formed with two mirrors of $1-\mathrm{m}$ radius of curvature. The mean cavity length was $108 \mu \mathrm{m}$ and its finesse was measured 217000 at the Cesium (Cs) D2 wavelength $(852 \mathrm{~nm})$. The cavity decay rate $(\mathrm{HWHM}) \kappa / 2 \pi \approx 3.2 \mathrm{MHz}$ while the dipole decay rate $\gamma_{\perp} / 2 \pi \cong 2.6 \mathrm{MHz}$ for the $\mathrm{Cs} 6 \mathrm{P}_{3 / 2}$ level. The atom-field coupling coefficient $g$ varies spatially, depending on the position of the atom with respect to the standing-wave structure of the intracavity field. The optimum value $g_{0} / 2 \pi$ was $\sim 11 \mathrm{MHz}$ for $\sigma_{ \pm}$transitions $\left[6 \mathrm{~S}_{1 / 2}\left(\mathrm{~F}=4, \mathrm{~m}_{\mathrm{F}}= \pm 4\right)\right.$ $\left.\rightarrow 6 \mathrm{P}_{3 / 2}\left(\mathrm{~F}=5, \mathrm{~m}_{\mathrm{F}}= \pm 5\right)\right]$ and $\sim 6 \mathrm{MHz}$ for $\pi$ transitions $\left[6 \mathrm{~S}_{1 / 2}\left(\mathrm{~F}=4, \mathrm{~m}_{\mathrm{F}}=0\right) \rightarrow 6 \mathrm{P}_{3 / 2}\left(\mathrm{~F}=5, \mathrm{~m}_{\mathrm{F}}=0\right)\right]$. Cold $\mathrm{Cs}$ atoms were provided from a standard magnetooptic trap (MOT), which was located $7 \mathrm{~mm}$ above the cavity. The trap was typically loaded for about $0.5 \mathrm{~s}$ before dropping atoms by quickly turning off the trapping beams and the magnetic field. Individual transit events typically lasted for $\sim 250 \mu$ s. The trapping beam power was varied to control the number of atoms falling through the cavity. The repumping beam was left on all the time so that falling atoms would be shelved in the $\mathrm{F}=4$ ground hyperfine level before entering the cavity. 
No spin polarization was performed on atoms to prepare for a specific Zeeman sublevel.

In hopes of eventually reaching the standard quantum limit for monitoring the position of a free mass as well as observing quantum measurement backaction, we were obliged to use a probe field detuned from the atomic resonance [8], [9]. The experimental protocol then involved determination of both the phase and amplitude of the cavity-output field with shot-noise-limited accuracy at a reasonable bandwidth. These quadrature amplitude (QA) signals provide a direct record of the complete time-evolution of the interaction energy $\left(E_{\text {int }}=\right.$ $\hbar g$ ) between the atom and cavity. In this dispersive regime we minimize not only the random heating generated by onresonance probing of the atomic motion [10] but also the information loss through the atomic decay to free space. We thus need a probe field void of any frequency noise while still arbitrarily tunable with respect to the atomic resonance. The cavity resonance is then locked tightly on the probe frequency to maintain a stable empty-cavity field and to avoid technical noise in the intrinsically frequency (phase)discriminating cavity transmission.

The probe beam was provided by a $\mathrm{Ti}$ : Sapphire laser with its fast linewidth narrowed by a prestabilizing reference resonator, which was in turn locked on the Cs resonance. The probe field was one of the $\mathrm{Ti}$ : Sapphire sidebands generated by an in-line electro-optic modulator (EOM) for tuning of the probe frequency. The probe field was too weak to establish a direct frequency lock to the cavity. On resonance, the saturation intracavity photon number for the QED cavity is $m_{0}=\gamma_{\perp}^{2} / 2 g_{0}^{2} \approx 0.1$, which sets a cavity throughput of $4 \pi \kappa m_{0} \approx 1 \mathrm{pW}$. Tuning off resonance would allow the probe power to be proportionally larger, however, it would still be extremely difficult to obtain a high-quality error signal for cavity locking. We thus used an auxiliary diode laser to stabilize the cavity length on a different longitudinal mode than the probe. The diode laser was detuned $16 \mathrm{~nm}$ off the Cs resonance and its power of $\sim 40 \mathrm{nW}$ through the cavity incurred an ac Stark shift of only $\sim 60 \mathrm{kHz}$ for the Cs resonance. The diode laser itself was stabilized by the same reference resonator shared with the $\mathrm{Ti}$ : Sapphire laser. Frequency detuning between the diode laser and the QED cavity was again furnished by a second EOM. (The cavity was locked on an RF sideband of the diode laser.) While this setup allowed us to have variable detunings among the atomic, cavity, and probe frequencies, in the experiment we kept a zero-detuning between the cavity and probe.

We used a balanced-heterodyne setup in order to achieve high-efficiency, zero-background photodetection of $\sim 1 \mathrm{pW}$ level of cavity transmission. The mode-cleaned and intensitystabilized local oscillator was derived from the same laser used for the probe beam with an appropriate frequency offset to avoid electronic noise pickup. The overall heterodyne efficiency $(\sim 0.32)$ was carefully measured in order to deduce the intracavity photon number and make quantitative comparisons with simulations. The difference photocurrent from the balanced heterodyne detectors was $0^{\circ}$-split to two identical copies, which were mixed with the in-phase and quadrature components of an additional RF local oscillator to produce an orthogonal pair of QA signals at baseband. The QA signals were filtered and digitized with a 12-bit resolution at a sampling rate of $10 \mathrm{MHz} /$ channel. Fluctuations of optical phases caused by vibrational disturbances along the beam propagation line were limited to frequencies below $\sim 1 \mathrm{kHz}$ and the recorded signal bandwidth typically extended from 1 to $300 \mathrm{kHz}$. Fortunately, this covered the dominant rates of variation in $E_{i n t}$, as the mean duration of individual transit events was $250 \mu \mathrm{s}$.

Fig. 1 shows the first measurement of the real-time evolution of the complex field amplitude brought by single atom motion. Dynamical variations in both QA's (at $300 \mathrm{kHz}$ bandwidth) are displayed versus time during transit events. Detunings $\Delta$ (atomic resonance-probe frequency) and probe powers (intracavity photon number $m$ with no atoms in cavity) are indicated on the figure. Note we have displaced the amplitude quadrature signals (upper traces) by +400 to prevent overlapping between the two traces. Although at times the transit signals seem to show internal structures, we still have yet to devise a clean way of distinguishing between variations caused by atomic motion through the spatial structure (both longitudinal and transverse) and/or optical pumping among atomic internal (Zeeman) states. The transition to the dispersive measurement regime is evidenced by the significant phase shift of the probe field and the fact that signals primarily reside in the phase quadrature at large detunings. The full-signal (combining both QA's) to rms-noise ratio (SNR) is estimated at 4.5 at $300 \mathrm{kHz}$ bandwidth, implying a relative sensitivity of $\sim 4 \times$ $10^{-4} / \sqrt{\mathrm{Hz}}$. This SNR lies only a factor of $\sim 2.7$ above the fundamental quantum noise level, which is estimated based on an ideal $100 \%$ quantum efficiency. Assuming that the largest signals correspond to atoms reaching the maximal coupling strength of $g_{0} / 2 \pi=11 \mathrm{MHz}$, this sets our broadband sensitivity to time-variations in $E_{\text {int }}$ to be $\sim 4.5 \mathrm{kHz} / \sqrt{\mathrm{Hz}}$. Considering the standing-wave structure of the cavity eigenmode, our data should in principle display a position sensitivity of $1.5 \times$ $10^{-10} \mathrm{~m} / \sqrt{\mathrm{Hz}}$ to atomic displacements along the cavity axis.

The atomic-transit signals can also be displayed in parametric plots of amplitude versus phase to examine the correlation induced between these two quantities by the atom-cavity interaction. This type of parametric plot is dictated by the interaction Hamiltonian for the atom and cavity mode. With fixed values of power $(\mathrm{m})$ and detuning $(\Delta)$, the only varying parameter in the plot is the atom-cavity coupling $g$, in the range of $\left[0, g_{0}\right]$. It is no longer relevant which underlying process (internal or external) controls the change of $g$. From this perspective the comparison between theory and experiment is thus simplified. Fig. 2(a) shows three transit phasors taken at different probe detunings, and illustrates transition from absorptive to dispersive regime. The data in each subplot (in dots, from two individual transit events) overlay theoretical predictions based on quantum and semiclassical theories. The point of no interaction $(g=0)$ is marked by a triangle. The $g=g_{0}$ endpoint is marked by a circle (o) for quantum theory and by a cross $(x)$ for semiclassical theory [12]. Fig. 2(b) illustrates the differences between the two theories regarding the nonlinear optical response of the atom-cavity system. The two transit phasors are shown for a fixed detuning but different 

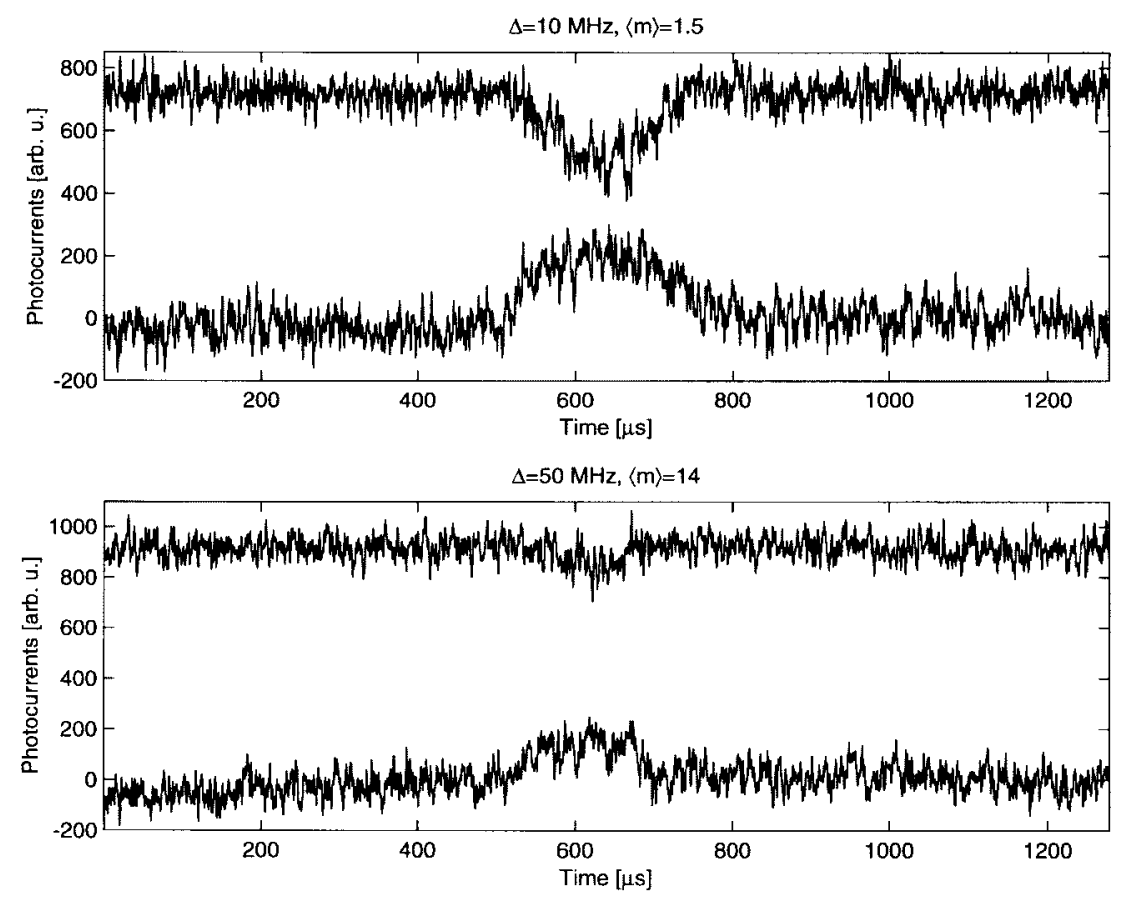

Fig. 1. Individual atom-transit signals recorded with a $300 \mathrm{kHz}$ bandwidth, with the atom-probe detuning $\Delta$ and probe power $<\mathrm{m}>$ (intracavity photon number in absence of atoms). Upper (lower) traces represent the amplitude (phase) quadrature signals. Upper traces are displaced by an additional +400 for clarity.

probe powers. The data clearly support the quantum theory which shows a discrepancy from the semiclassical theory. We stress that our experiments allow exploration of nonlinear quantum dynamics at single quantum realizations, instead of from ensemble averages [11], [12].

\section{Photon-Covalent Binding of Atom And CAVity}

In the second experiment, a very small cavity of length 10.1 $\mu \mathrm{m}$ and finesse 180000 provided us with the largest coupling $g_{0}$ in a cavity QED system to date. The relevant rates were $\left(g_{0}, \kappa, \gamma_{\perp}, T^{-1}\right) / 2 \pi=(120,40,2.6,0.002) \mathrm{MHz}$. These rates correspond to critical photon and atom numbers $\left(m_{0} \equiv\right.$ $\left.\gamma_{\perp}^{2} / 2 g_{0}^{2} ; \quad N_{0} \equiv 2 \kappa \gamma_{\perp} / g_{0}^{2}\right)=\left(2.3 \times 10^{-4} ; 0.015\right)[1] . \mathrm{Cs}$ atoms cooled to $\sim 20 \mu \mathrm{K}$ were again dropped down through the cavity. Here the frequency detuning between the atomic and empty-cavity resonances remained zero $\left(v_{\text {atom }}=v_{\text {cavity }}=\right.$ $v_{0}$ ). The cavity length was stabilized by a chopped auxiliary beam tuned on Cs resonance. A freely-tunable probe beam was used to explore the eigenspectrum of the atom-cavity system in cavity transmission, again with balanced heterodyne detection.

As an atom falls into the cavity, the increasing $E_{\text {int }}=\hbar g(\vec{r})$ causes the otherwise coincident atomic and cavity resonances to split into two "vacuum-Rabi" normal modes, located at $v_{0} \pm g(\vec{r}) / 2 \pi$, corresponding to two dressed states of the atom-cavity system. We can now watch this mode-splitting process in real time. For the few atoms that actually reach a region of optimal coupling as they fall through the cavity, the vacuum-Rabi sidebands should be swept outward (from the cavity resonance) in frequency to a maximum of $\pm g_{0} / 2 \pi=$ $\pm 120 \mathrm{MHz}$. Therefore, for the probe beam with a fixed frequency detuning at $\Delta=v_{0}-v_{\text {probe }}= \pm 120 \mathrm{MHz}$, the cavity transmission will show the largest increase. Similarly, for the probe beam of $\Delta=0$, the cavity transmission

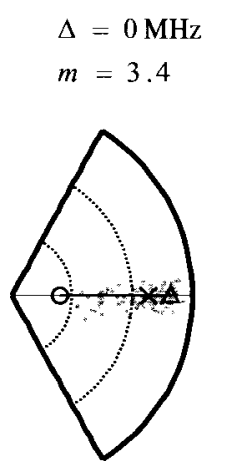

$$
\begin{aligned}
\Delta & =10 \mathrm{MHz} \\
m & =2.8
\end{aligned}
$$$$
\Delta=50 \mathrm{MHz}
$$$$
m=9
$$
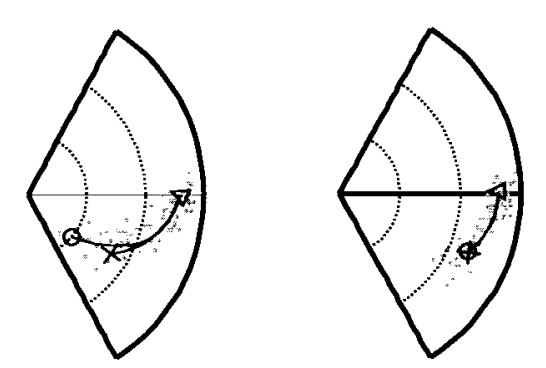

(a)

$\Delta=10 \mathrm{MHz}$
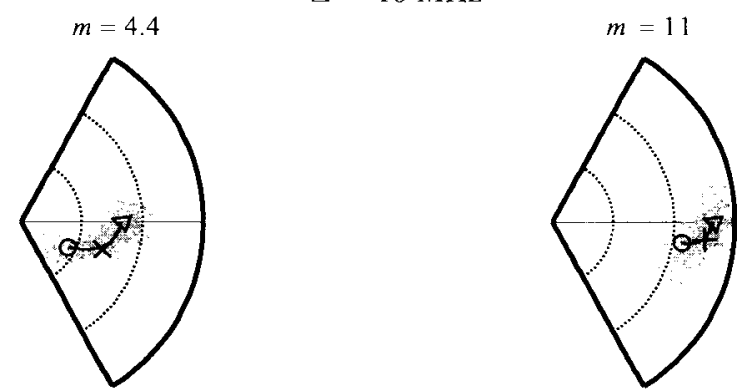

(b)

Fig. 2. (a) Dependence of transit phasor shapes on detuning. Each subplot shows an overlay of two transit data (dots), master equation-based quantum theory (solid curve ending in circle), and semiclassical theory (solid curve ending in $X$ ). (b) Nonlinear response of the transit phasor, with a fixed detuning $(10 \mathrm{MHz})$ and different probe powers, indicated by the mean intracavity photon number in each case.

shows the largest attenuation. For atoms that do not achieve optimal coupling, we should expect the maximum increase of cavity transmission to occur at some intermediate detunings 


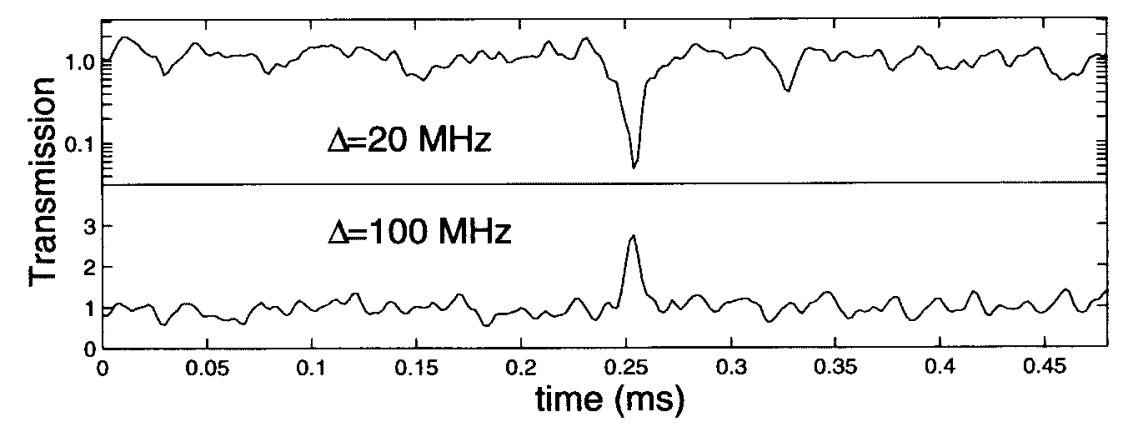

Fig. 3. Simultaneous recording of two normalized probe transmissions through the atom-cavity system. Upper trace, $\Delta \approx 0 ;$ lower trace, $\Delta \approx 120$ MHz. Bandwidth is $100 \mathrm{kHz}$.

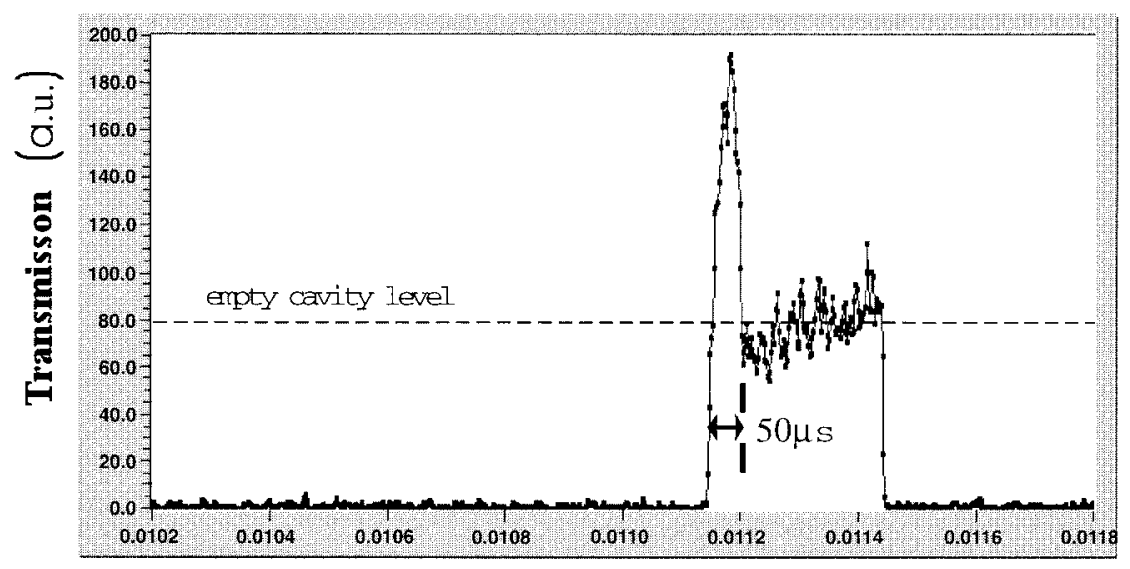

time (s)

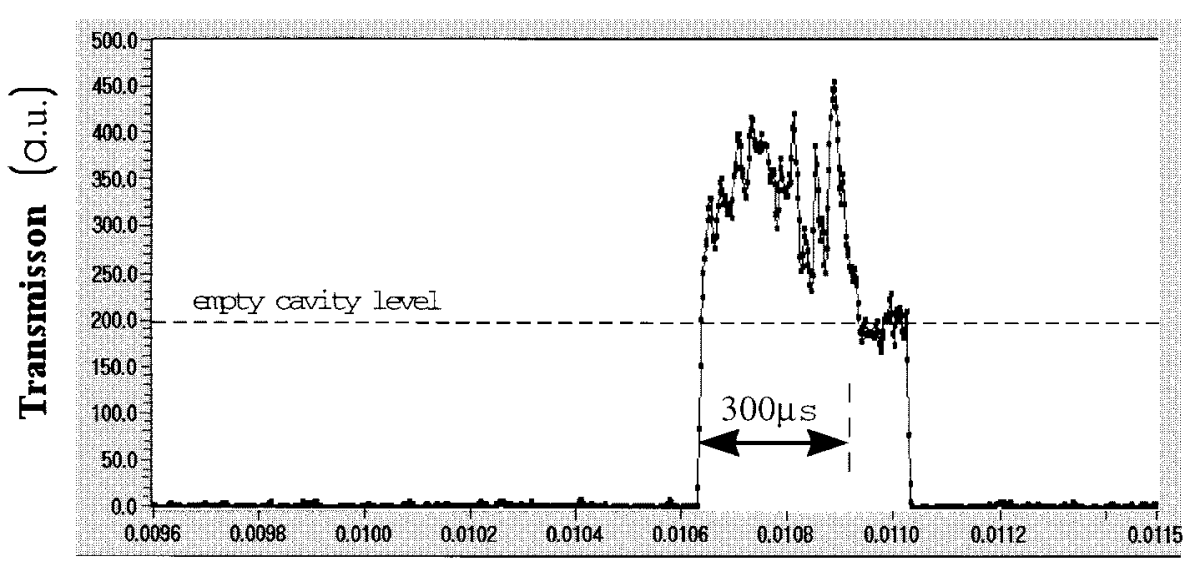

time (s)

Fig. 4. Strong-coupling induced mechanical effects on atoms. In (b), a probe beam at $\Delta \sim g_{0}$ is triggered on by the entrance of an atom into the cavity. The atomic transit time is extended to $\sim 300 \mu \mathrm{s}$, compared with the $100 \mu \mathrm{s}$ normal duration in the absence of the trapping beam, as in (a).

between $40 \mathrm{MHz}$ and $100 \mathrm{MHz}$. Fig. 3 shows an example of real-time changes in transmission of the atom-cavity system produced by an individual atom transit. The transmission of two probe beams are simultaneously recorded. In the plot the transmissions are normalized against the empty-cavity values. For the probe near resonance $(\Delta \approx 20 \mathrm{MHz})$, the cavity transmission decreases as an atom falls through the cavity. At the same time, the cavity transmission increases as the lower Rabi sideband for the second probe tuned to $\Delta \approx 100 \mathrm{MHz}$. We also note that the signal contrasts for two probes are lower than for single-probe measurements due to system saturation.
Carrying out repeatedly the transmission measurement, the spectrum of the atom-cavity system can be mapped out with a frequency sweep on a single probe. While the double-peaked structure of the vacuum-Rabi splitting is clear, with peaks near $\pm g_{0} / 2 \pi$, it is equally clear that the spectrum showed some asymmetry between red and blue probe detunings (see [7, Fig. 4]). Since the red (blue) Rabi-sideband corresponds to the lower (upper) dressed state, the associated attractive (repulsive) mechanical light force is expected to affect an atom's CM motion. Indeed, weak excitation by a coherent probe tuned to $\Delta_{ \pm} \approx \pm g_{0}$ gives rise to a pseudopotential (for 
times $\gg \kappa^{-1} \sim 4 \mathrm{~ns}$ ), with depth on the order of $\mp \hbar g_{0} P_{\mp}$ [13], with $P_{\mp}$ the excitation probability of the relevant dressed state. Since $\hbar g_{0} / \kappa_{B} \approx 7 \mathrm{mK}$, such light forces can be significant even with a cavity of less than 1 photon, given that atoms are precooled and many Rabi-cycles occur during the transit time. Therefore, a blue-detuned probe $(\Delta<0)$ creates spatially-varying potential barriers and prevents atoms from reaching areas of optimal coupling. On the other hand, a red-detuned probe $(\Delta>0)$ will coerce atoms to channel into regions of high intensity and strong coupling. This attractive potential can be used as a trap for single atoms. In this context, the quantity $\hbar g_{0}$ may be interpreted as a covalent atom-cavity binding energy associated with the oscillatory exchange of a single photon.

Experimentally, a weak probe beam tuned near the emptycavity resonance is used to sense the arrival of an atom to the cavity. A drop in transmission of this sensing beam then triggers off this resonant field and triggers on a second field at $\Delta \approx g_{0}$ to create the trapping potential. In Fig. 4 , we show an example of an atom that has been held in the cavity field for more than $300 \mu \mathrm{s}$ (b), whereas the longest possible transit times observed without the strong-coupling-induced mechanical effects are $\sim 100 \mu$ s (a).

\section{CONClusion AND Future OUtLOOK}

Combining cold Cs atoms with an optical cavity capable of inducing strong coupling, we have recorded the real-time evolution of the complex field amplitude of cavity transmission with a large bandwidth and nearly quantum noise-limited signal-to-noise ratio. Phasor diagrams built upon one or just a few single-atom transits explore the strong-coupling-induced correlation between two quadratures of the field and reveal the quantum nature of the atom-cavity interaction via the signature of system saturation. Detection in the dispersive regime allows us to map out a single-atom trajectory in the cavity in a nondestructive manner. With a large coherent coupling rate $g_{0}$, system dynamics such as vacuum-Rabi splitting are monitored in real time with single atoms falling through the cavity. Mechanical effects on single atom CM motion through atomcavity strong couplings have also been demonstrated. Trapping of single atoms in the cavity quantum field is being explored.

The demonstrated experimental regime of measurement sensitivity and bandwidth should allow us in future cavity QED research to observe detailed atomic CM trajectories. With future additional capabilities of being able to manipulate atomic $\mathrm{CM}$ in real time, we anticipate progress in the investigation of feedback control of quantized atomic CM motion. Quantitative experiments on conditional quantum dynamics [14] should also become possible as improvement on the measurement accuracy will shorten the overall observation time before measurement back action kicks in. Toward this goal, we are working to trap and localize atoms within the cavity and investigate the interplay between the external degree of freedom (quantized atomic CM motion) and the internal degree of freedom of the composite atom-cavity "molecule" [15].

\section{ACKNOWLEDGMENT}

The authors wish to acknowledge M. S. Champan's vital contribution to the project.

\section{REFERENCES}

[1] H. J. Kimble, "Structure and dynamics in cavity quantum electrodynamics," in Cavity Quantum Electrodynamics, P. R. Berman, Ed. San Diego, CA: Academic, 1994, pp. 203-266.

[2] H. M. Wiseman, "Quantum theory of optical feedback via homodyne detection," Phys. Rev. Lett., vol. 70, p. 548, 1993.

[3] H. Mabuchi, Q. A. Turchette, M. S. Chapman, and H. J. Kimble, "Realtime detection of individual atoms falling through a high-finesse optical cavity," Opt. Lett., vol. 21, pp. 1393-1395, 1996.

[4] H. Mabuchi, J. Ye, and H. J. Kimble, "Full observation of single-atom dynamics in cavity QED," submitted for publication.

[5] C. M. Caves and G. J. Milburn, "Quantum-mechanical model for continuous position measurements," Phys. Rev. A, vol. 36, p. 5543, 1987.

[6] H. M. Wiseman, "Quantum trajectories and quantum measurement theory," Quant. Semiclass. Opt., vol. 8, p. 205, 1996.

[7] C. J. Hood, M. S. Chapman, T. W. Lynn, and H. J. Kimble, "Real-time cavity QED with single atoms," Phys. Rev. Lett., vol. 80, p. 4157, 1998.

[8] P. Storey, T. Sleator, M. Collett, and D. Walls, "Contractive states of a free atom," Phys. Rev. A, vol. 49, p. 2322, 1994.

[9] M. A. M. Marte and P. Zoller, "Quantum nondemolition measurement of transverse atomic position in Kapitza-Dirac atomic beam scattering," Appl. Phys. B, vol. 54, p. 477, 1992.

[10] A. C. Doherty, A. S. Parkins, S. M. Tan, and D. F. Walls, "Motion of a two-level atom in an optical cavity," Phys. Rev. A, vol. 56, p. 833, 1997.

[11] M. Brune, F. Schmidt-Kaler, A. Maali, J. Dreyer, E. Hagley, J. M. Raimond, and S. Haroche, "Quantum Rabi oscillation: A direct test of field quantization in a cavity," Phys. Rev. Lett., vol. 76, p. 1800, 1996.

[12] R. J. Thompson, Q. A. Turchette, O. Carnal, and H. J. Kimble, "Nonlinear spectroscopy in the strong-coupling regime of cavity QED," Phys. Rev. A, vol. 57, p. 3084, 1998.

[13] A. S. Parkins, private communications, 1996

[14] H. Mabuchi, "Standard quantum limits for broadband position measurement," Phys. Rev. A, vol. 58, p. 123, 1998.

[15] D. W. Vernooy and H. J. Kimble, "Well-dressed states for wave-packet dynamics in cavity QED,” Phys. Rev. A, vol. 56, p. 4287, 1997.

Jun Ye, for photograph and biography, see this issue, p. 536.

Christina J. Hood, photograph and biography not available at the time of publication.

Theresa Lynn, photograph and biography not available at the time of publication.

Hideo Mabuchi, photograph and biography not available at the time of publication.

David W. Vernooy, photograph and biography not available at the time of publication.

H. Jeff Kimble, photograph and biography not available at the time of publication. 